\title{
IoT Based Ticket Checking System
}

\author{
Kirti Dhiman ${ }^{1}$, Er. CK Raina ${ }^{2}$ \\ Department of Computer Engineering, Adesh Institute of Technology Gharuan, India ${ }^{1}$ \\ HOD, Department of Computer Engineering, Adesh Institute of Technology Gharuan, India ${ }^{2}$
}

\begin{abstract}
Internet of things. The term Internet of Things was used by Kevin Ashton in 1999. IOT is like a vehicle used to as a "Smart Devices" and other items like Electronics, softwares.IOT words was Invented from a two words "Internet "and "Things". Internet is a vast network. The Internet is the global system of interconnected computer networks that use the protocol to link devices. Internet is used in daily life to communicate, search information etc. things means important information or devices. In recent days a population is gained day by a day and smart cities have gained popularity. In this paper we present a "IOT BASED TICKET CHECKING SYSTEM". This system is consist of an IOT module that is used to check the tickets of passenger in trains. This system describes the whole architecture of a train system.
\end{abstract}

Keywords: IOT, Passengers, Smart cities, Smart transport, Ticket checking, passengers, safety.

\section{INTRODUCTION}

IOT is basically used to connect the several devices. IOT words was Invented from a two words "Internet" and "Things". Internet is a vast network. The Internet is the global system interconnected computer networks that use the protocol to link devices. Things means important information or devices.IOT refers

or used objects that is part of the internet the most consequences of IOT is the generation of huge quantities of data.IOT is implemented for large scale data that need encoded 50 to 100 trillion data. We represent an overview of internet of things. We present a "IOT BASED TICKET CHECKING SYSTEM". This system increasing public awareness about the changing system of infrastructure. Every human being is surrounded by 1000 to 5000 objects as per day[10]. Each of these objects are linked through a internet or network.IOT refers to a network of object. IOT is also known as a Distributed Network.IOT is the network of physical objects-devices, vehicles, buildings and other items embedded with electronics, software, ser ors, and network connec ${ }^{~}{ }^{i}$ - that enables th Jbjerrs tr sollect and exchangin。 ...ta [1]. re ar veral reaso to change the system:

- In trains, there is several people travel without purchasing the tickets.

- Due to crime like raped, train murder, chain snatching.

- To save the money. For example. manpower

- Save the time.

The Internet of Things involves an increasing number of smart interconnected devices and sensors (e.g. cameras, biometric and medical sensors) that are often nonintrusive, transparent and invisible. IOT will encourage the development of new applications such as smart cities, grids, and transportation systems. During the integration, QOS and QOE, as well as data security, privacy, and reliability, are considered as the critical concerns. The integration of cloud computing and IOT demands for high quality for these type of issues [3]. Each thing is uniquely identifiable through its embedded computing system but is able to interoperate within the existing Internet Infrastructure. Experts estimate that the IOT will consist of almost 50 billion objects by 2020[11].

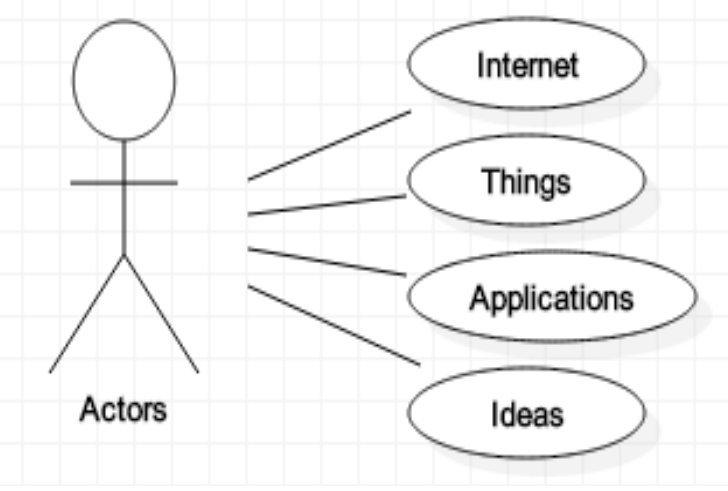

Fig-1 IOT
Fig-1 describes that one actor use internet and some application to gain some knowledge and requires any new ideas.

\section{Application}

Several applications can benefit from the availability of social relationships between things interconnected to a network composed of trillions of nodes [4]. While a few interesting applications can be the increase in the number and categories of objects able to connect to the Internet [4].

In fig-2, people will be able to connect to the Internet in many ways. Today, most people connect to the Internet through some of devices such as PCs, tablets, TVs, and Smartphone's and social networks like whatsapp, facebook, twitter, LinkedIn. 


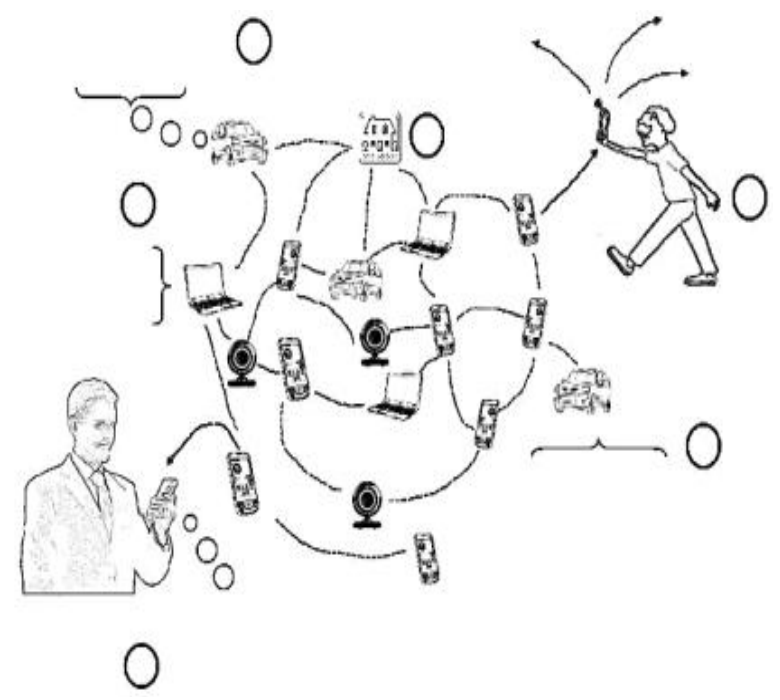

Fig-2[4]

\section{Internet}

As a daily routine we called internet as a net. Net means a number of networks that connects in all over the world.

\section{Things}

Things mean an important information or data. Things are a mixture of hardware, software, etc. With IOT, devices typically gather data and stream it over the Internet to a central source, where it is analyzed and processed [12].

\section{IOT Architecture}

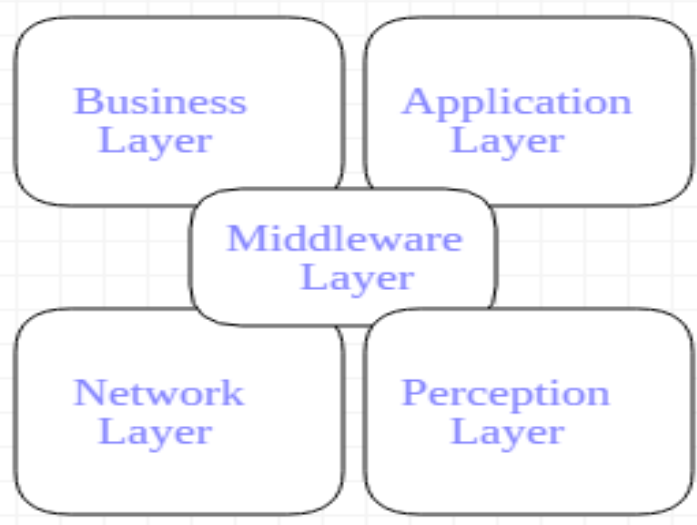

Fig-3 architecture of IOT [3]

Layer 1, the Business layer represent all the responsibility for all layers. It represents the IOT sense and directly deal with objects. It is also called sensing layer. It defines an additional base station [5].

Layer 2, the application layer is also called transport layer. It defines" how to transfer the data over a network".

Layer 3, the middleware layer is also called a application support layer [5].it stores analyses and processes the information related to items received from layer 2[5]. This is the layer where the IOT runs[5].

Layer 4, the network layer is also called a gateway layer. It is used to deliver a packet from source to destination.
Layer 5, the perception layer is also called a sensing layer. The perception layer can be abstracted as sensing components/nodes, actuator. Sensing components include sensors, RFID, bar code label, intelligent detection instruments and meters, etc [5].

\section{Objectives}

- Easier and safe

- IOT represent future trends for smart transportation

- Security for remove crimes.

- Representing human behavior after changing system.

- This scenario is not dependent from each other.

- Self organization.

- Freed from a big line

- Time saves

\section{RELATED WORKS}

Indian Railway is the largest transport all over the India. In this Indian railways have a ticket checker that is known as a palmtop. Palmtop is connected to central server. From the Palmtop TTE update the passenger's presence in the train to central server [11].

But here we present a ticket checker System that is organized in a sleeper coach. Therefore the communication technology which is proposed to use for proper implementation of this assignment is based on that communication technology which are currently used in real time movements of trains in Satellite Imaging Rail Navigation [11].

\section{PROPOSED WORK}

1. We make a system that checks the ticket of passenger in trains through IOT based System.

2. After entered in train ticket is checked by a System. Or we can say that passenger give the tickets to system to entered next.

3. If anybody can not purchase ticket on tickets counter then a passengers can buy a ticket from this system.

4. So this system is safe from crime and save the time of passenger.

5. Passenger checked his/her ticket by scan the barcode of ticket into the system.

6. System is used in sleeper trains.

7. Checking In And checking Out System

8. TICKET VALIDATION: In this module where when the user buys the ticket, the source point destination geopoints, ticket type, expiry time \& date.

9. TICKET BUYING

The user selects source, destination, class, no. of Adult and child tickets, ticket type like return or single etc [12].

Fig-4 describes the system to enter in the train. First of all a people checked his/her ticket by bar code if accepted the enter the next. Represent if passenger have not ticket the passenger can buy the ticket from this system. 


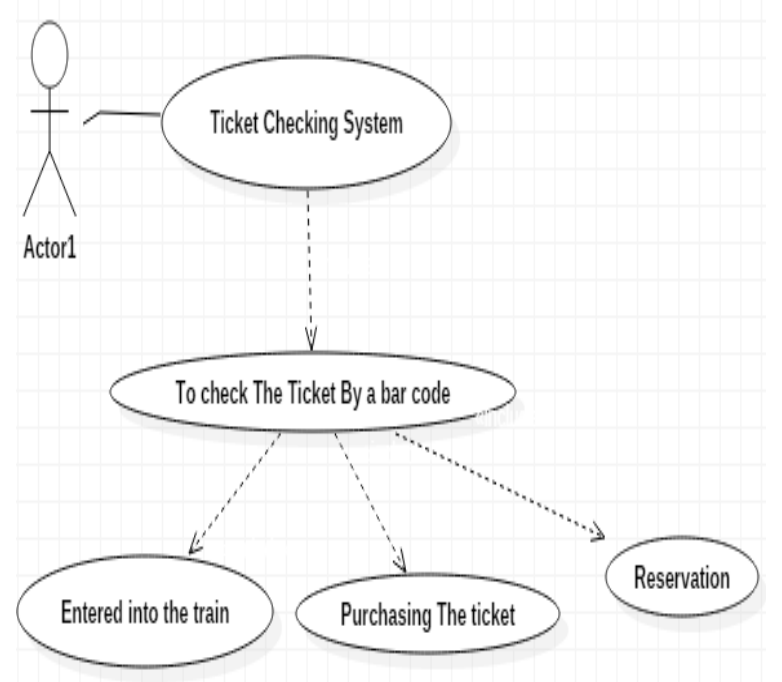

Fig-4

Bar code:

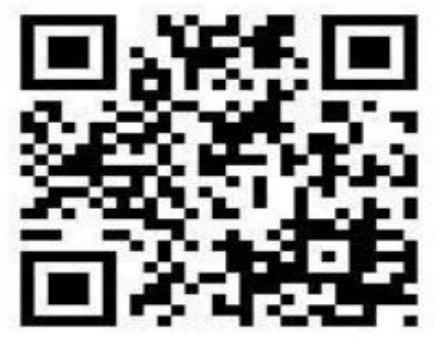

Fig-5

\section{Flow chart}

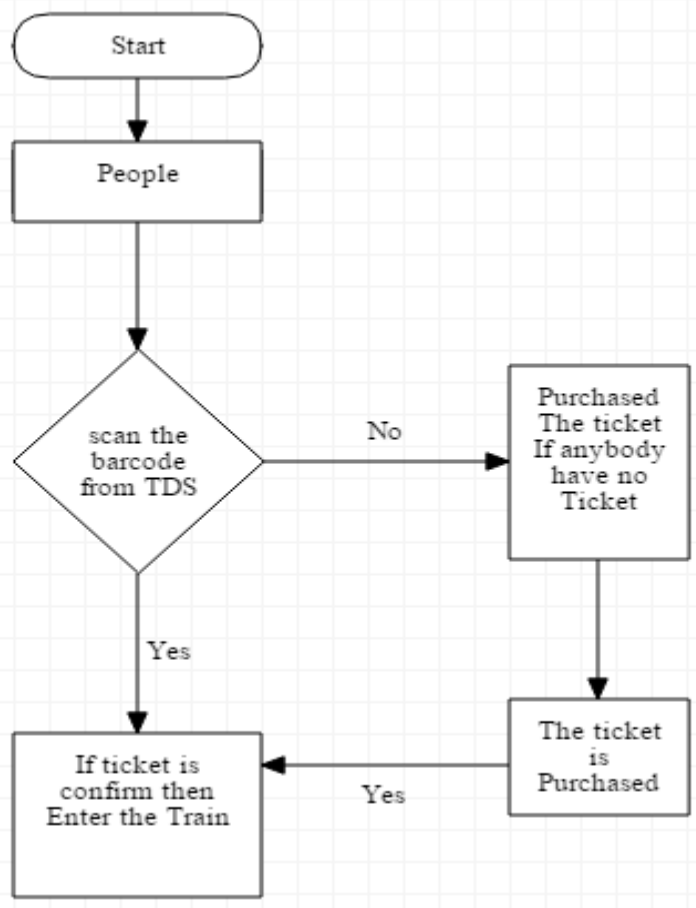

Fig-6
Future opportunities and challenges

Security: IOT connects more devices and transfers the entry points for malware. Less expensive devices are more subject to tampering [6].these challenges will become more pressing as use of the physical Web continuous to grow [7].when we apply this system in train then our world is crime free.

\section{Actions and control}

IOT provides an action to control the physical world. Many modern systems benefit from remote control because it simplifies a physical interaction design and extend capability [7].

\section{Complexity, Confusion and Integration Issue}

The IOT system with multiple platforms, numerous protocols and large numbers of APIs lacks in integration and testing which leads to confusion around evolving standards [6].

\section{Design Smart Trains}

IOT can be used to design smart trains. For example change management, security, chain snatching system.

\section{Smart City}

We don't want to write about it this topic and there are a enough articles in place to learn more about. Keeping city connected will help in many ways and make people more productive [8].

\section{Smart Industry}

It applies to all industry that exists today, you can think of a new use-case everyday to jump in Internet of Things world. It's quite common for every industry [8].

\section{Manageability}

Easy to maintain the infrastructure.

\section{Extensibility}

New applications require only endpoints and application programming interfaces (APIs) to integrate with the existing infrastructure [2].

\section{Flexibility}

The architecture allows city managers and citizens to utilize the same services and information for their specific needs [2].

\section{CONCLUSION}

In this paper we represent main layered architecture of IOT. We make a system that checks the ticket of passenger in trains through IOT based System. After entered in train ticket is checked by a System. We studied the entire layer that is used in IOT. This system describes the whole architecture of IOT. In fact, the most IOT architecture includes all the existing technologies that are used to make a new System. 


\section{REFERENCES}

1. Gubbi, J., Buyya, R., Marusic, S., \& Palaniswami, M. (2013). Internet of Things (IoT): A vision, architectural elements, and future directions. Future generation computer systems, 29(7), 16451660 .

2. S.Geetha M Phil. Research Scholar, Department of Computer Science, Kovai Kalaimagal College of Arts and Science, Coimbatore, India." Social Internet of Things".

3. Niclota-Cristina Gaitan, Vasile Gheorghita Gaitan,Ioan Ungurean Ungurean ;"A Survey on the Internet of things software Architecture". International journal of advanced CSE Dec-2015.

4. G. Bhavani1, *, S. Sangeetha2, S. Sivakumari3." Protocols and Challenging Issues in IoT".

5. Roy Want, Bill N. Schilit, and Scott Jenson, Google.”Enabling the iot".

6. Assoc. Prof. DimiterG. Velev, PhD." internet of things Analysis and Challenges".

7. Yadav, Omprakash, et al. "Online Reservation System Using QR Code based Android Application System." ISSN 225 (2007): 03153.

8. Sandakan, Neha, Rane Dipti, and Sachin Pandey. "2013 Android Railway Ticketing with GPS as Ticket Checker." Proceedings of National Conference on New Horizons In IT-NCNHIT. 2013.

9. Ortiz, A. M., Hussein, D., Park, S., Han, S. N., \& Crespi, N. (2014). The cluster between internet of things and social networks: Review and research challenges. IEEE Internet of Things Journal, 1(3), 206-215.

10. Xia, F., Yang, L.T., Wang, L. and Vinel, A.,2012.Internet of things. International Journal of Communication Systems, 25(9), p.1101.

11. Yun, M., \& Yuxin, B. (2010, June). Research on the architecture and key technology of Internet of Things (IoT) applied on smart grid. In Advances in Energy Engineering (ICAEE), 2010 International Conference on (pp. 69-72). IEEE. 